\title{
Enhancement of solubility of dexibuprofen applying mixed hydrotropic solubilization technique
}

\author{
Boushra Mohamed El-Houssieny ${ }^{1}$, Esmat Zein El-Dein ${ }^{2}$, Hussien Mohamed El-Messiry ${ }^{1, *}$ \\ ${ }^{1}$ Department of Pharmaceutics, National Organization of Drug Control and Research, Giza, Egypt; \\ ${ }^{2}$ Department of Pharmaceutical Technology, Faculty of Pharmacy, Tanta University, Tanta, Egypt.
}

\begin{abstract}
Summary Dexibuprofen, is a practically water-insoluble nonsterodial anti-inflammatory drug which has a better anti-inflammatory effect than ibuprofen. A mixed hydrotropic solubilization technique was applied in order to improve the aqueous solubility and dissolution rate of dexibuprofen. Nine formulae were prepared using different concentrations of hydrotropic agents (sodium citrate dihydrate and urea). The prepared formulae were inspected visually for color and odor. Hygroscopicity, micromeretic properties, solubility, and $\mathrm{pH}$ for $1 \%$ aqueous solutions were determined. In-vitro dissolution studies of the different prepared formulae were performed adopting the USP XXII dissolution method type I basket apparatus method. The prepared formulae were characterized by infrared (IR) spectroscopy and differential scanning calorimetry (DSC). The prepared formulae were a white color, odorless, slightly hygroscopic and exhibited good flow properties. Formulae containing higher amounts of hydrotropic agents exhibited an increase in the pH, solubility, rate and amount of dexibuprofen released from the dissolution medium. The highest dissolution rate was achieved from the $\mathbf{F 9}$ formula at drug:sodium citrate dihydrate:urea ratio (1:3:7.5). IR and DSC thermograph of dexibuprofen, hydrotropic agents and prepared formulae indicated the presence of intermolecular interaction between drug and hydrotropic agents which increased solubility and dissolution rate of drug, also, there is no chemical interaction confirming the stability of the drug with hydrotropic agents.
\end{abstract}

Keywords: Solubility, dexibuprofen, hydrotropic agents, formulae

\section{Introduction}

Dexibuprofen $((\mathrm{S})-(+)$-ibuprofen), which has better anti-inflammatory effects than ibuprofen and less gastric damage belongs to class II of the Biopharmaceutical Classification System (BCS) having low water solubility which is the rate limiting step in absorption of drug $(1,2)$.

Poor solubility manifests many in vivo limitations like incomplete release, poor bioavailability, food effects, and higher inter-subject variability. However, different efforts have been demonstrated to improve bioavailability by increasing dissolution rate, for example: formulation of solid dispersions, solid

*Address correspondence to:

Dr. Hussien Mohamed El Messiry, Department of Pharmaceutics, National Organization of Drug Control and Research, 51Wezaret El-Zeraa street, Dokki, Giza, Egypt.

E-mail: hussienelmessiry@hotmail.com solutions, micronization, nanosuspension, cocrystal molecular encapsulation with cyclodextrin, co-solvency, hydrotropy, spray drying, solubilization with surfactant, microemulsion, salt formation, polymorphism and combinations of effects (3).

Hydrotropy is the term originally put forward by Neuberg (4), to describe the increase in the solubility of a solute by the addition of fairly high concentrations of alkali metal salts of various organic acids. However, the term has been used in the literature to designate nonmicelle-forming substances, either liquids or solids, organic or inorganic, capable of solubilizing insoluble compounds. The hydrotropic solubilization process involves cooperative intermolecular interaction with several balancing molecular forces, rather than either a specific complexation event or a process dominated by a medium effect, such as co-solvency or saltingin. Hydrotropic agents have been observed to enhance the aqueous solubility of poorly water-soluble drugs 
$(5,6)$. The aim of our work is to prepare a soluble form of dexibuprofen which can be used in various dosage forms.

\section{Materials and Methods}

\subsection{Materials}

Dexibuprofen ((S)-(+)-ibuprofen) standard was purchased from Sigma-Aldrich (St. Louis, MO, USA). Dexibuprofen raw material was a gift from Future Pharmaceutical Industries (FPI) (Badr city, Cairo, Egypt). Distilled water was prepared in Central Nervous System (CNS) lab, National Organization of Drug Control and Research (NODCAR) (Dokki, Giza, Egypt). Methanol was purchased from Sigma-Aldrich (Taufkirchen, Germany). Sodium citrate dihydrate and urea were purchased from Medex (Medical Export Co Ltd, Near Naseby, Northamptonshire, UK).

\subsection{Equipment}

Analytical balance (Sartorius AG, Goettingen, Germany). Dissolution Test Station (Hanson Research, SR8 Plus, Chatsworth, USA). Drying Oven Digit (Raypa - R. Espinar, S.L., Barcelona, Spain). Differential scanning calorimetry (DSC) calorimeter (Shimadzu DSC-50, Nakagyo-ku, Kyoto, Japan). Hot plate and stirrer (Jenway, Dunmow, Essex, UK). Infrared spectrophotometer (IR Prestige-21, Shimadzu, Nakagyo-ku, Kyoto, Japan). pH meter (Jenway, Dunmow, Essex, UK). Ultrasonic water bath (Crest Ultrasonics Shanghai Ltd., Song Jiang, Shanghai, China). UV-VIS spectrophotometer (UV-2450, Shimadzu, Nakagyo-ku, Kyoto, Japan).

\subsection{Preparation of formulae}

Nine formulae were prepared using different concentrations of hydrotropic agents (sodium citrate dihydrate and urea) (Table 1). Urea and sodium citrate were accurately weighed. A minimum (possible) quantity of distilled water at $80-85^{\circ} \mathrm{C}$ contained in a 250 $\mathrm{mL}$ beaker was used to dissolve the urea and sodium citrate for quick dissolution. Then, dexibuprofen was

Table 1. Composition of the prepared formulae

\begin{tabular}{ll}
\hline Formula & Dexibuprofen:Sodium citrate:Urea \\
\hline F1 & $1: 1: 2.5$ \\
F2 & $1: 2: 2.5$ \\
F3 & $1: 3: 2.5$ \\
F4 & $1: 1: 5$ \\
F5 & $1: 2: 5$ \\
F6 & $1: 3: 5$ \\
F7 & $1: 1: 7.5$ \\
F8 & $1: 2: 7.5$ \\
F9 & $1: 3: 7.5$ \\
\hline
\end{tabular}

added at $30-40^{\circ} \mathrm{C}$ and a teflon coated magnetic bead was dropped in it. Stirring of magnetic bead in beaker was started using a magnetic stirrer, maintaining the temperature at $30-40^{\circ} \mathrm{C}$. Dexibuprofen was completely solubilized. Stirring was continued until a semisolid mass was obtained in the beaker (after evaporation of a large quantity of water). The semisolid mass obtained was spread on several watch glasses in thin layers for quick drying. The watch glasses were kept in an oven, maintained at $40^{\circ} \mathrm{C}$ for drying. When the mass became pulverizable, it was triturated with the help of mortar and pestle and again kept in oven for drying. After almost complete drying, the powder of the solid dispersion was passed through sieve \#100 and was kept for 6 days in a desiccator containing silica gel and stored in air-tight containers.

\subsection{Evaluation of the prepared formulae}

\subsubsection{Visual inspection}

The prepared formulae were inspected visually for color and odor.

\subsubsection{Determination of loading efficacy}

A weighed amount of each of the prepared formulae equivalent to a theoretical content of $100 \mathrm{mg}$ drug was accurately weighed and allowed to disintegrate completely in $100 \mathrm{~mL}$ distilled water, by sonicating for $20 \mathrm{~min}$ so as to dissolve drug and hydrotropic agents. One $\mathrm{mL}$ of each solution was diluted to $100 \mathrm{~mL}$ with distilled water, the absorbance of the solution was measured spectrophotometrically at $\lambda_{\max } 222 \mathrm{~nm}$ using distilled water as blank. The concentration of the drug was calculated. Each sample was assayed in triplicate and an average of three determinations was calculated.

\subsubsection{Solubility and $p H$}

Solubility was determined by taking an excess quantity of prepared formulae in fixed volumes of solvent (distilled water). The resulting solution was placed on a water bath sonicator for $24 \mathrm{~h}$. After equilibrium the samples were centrifuged and then supernatant was filtered through a $0.45 \mu \mathrm{m}$ filter membrane and the concentration of drug in the saturated solution in formulae under investigation was determined spectrophotometrically at $\lambda_{\max } 222 \mathrm{~nm}$ after appropriate dilution of the filtrate using distilled water as a blank(3). Each experiment was done in triplicateand an average of three determinations was calculated. The $\mathrm{pH}$ for $1 \%$ aqueous solution was determined.

\subsubsection{Hygroscopicity}

Hygroscopicity was measured for $10 \mathrm{~g}$ powder $\left(\mathrm{m}_{1}\right)$ 
stored on a watch glass at $25^{\circ} \mathrm{C}, 80 \%$ R.H. for $24 \mathrm{~h}$ and the mass gain $\left(\mathrm{m}_{2}\right)$ was recorded $(7)$. The percent mass gain $(\% \mathrm{~m} / \mathrm{m})$ was calculated from the following equation: $\left(\mathrm{m}_{2} / \mathrm{m}_{1}\right) \times 100$.

\subsubsection{In vitro dissolution studies}

In vitro dissolution studies of the different prepared formulae were performed adopting the USP XXII dissolution method type I basket apparatus method. The samples were separately encapsulated in transparent hard gelatin capsules. Five hundred $\mathrm{mL}$ of distilled water was used as dissolution media at $37 \pm 0.5^{\circ} \mathrm{C}$ maintaining stirring speed at $50 \mathrm{rpm}$. The samples were withdrawn at specified time intervals and replaced with an equivalent volume of fresh dissolution medium at different time intervals to keep the volume constant and estimated spectrophotometrically at $\lambda_{\max } 222 \mathrm{~nm}$ for dissolving drug using distilled water as a blank(8). All dissolution tests were performed in triplicate and an average of three determinations was calculated.

\subsubsection{Micromeritic properties}

The prepared formulae were characterized by their micromeretic properties such as bulk and tapped densities, Hausner ratio, \% compressability and angle of repose.

Determination of bulk and tapped densities: Five grams powder $(\mathrm{m})$ in $250 \mathrm{~mL}$ graduated cylinder and the volume occupied (bulk volume $\mathrm{v}_{\mathrm{b}}$ ) recorded. The graduated cylinder was tapped till constant volume was obtained and the volume of powder (true or tapped volume $\left.v_{t}\right)$ was then recorded. The bulk density $\left(P_{b}\right)$ and tapped density $\left(\mathrm{P}_{\mathrm{t}}\right)$ were calculated in $\mathrm{g} / \mathrm{mL}$ by dividing the weight over the corresponding volume. Hausner ratio and \% compressability (Carr's index) were calculated using measured values for bulk density $\left(\mathrm{P}_{\mathrm{b}}\right)$ and tapped density $\left(\mathrm{P}_{\mathrm{t}}\right)$ according to the following equations (9): Hausner ratio $=\mathrm{P}_{\mathrm{t}} / \mathrm{P}_{\mathrm{b}}, \mathrm{Ci} \%=$ $\left[\left(\mathrm{P}_{t}-\mathrm{P}_{\mathrm{b}}\right) / \mathrm{P}_{\mathrm{t}}\right] \times 100 . \mathrm{Ci} \%$ is the $\%$ compressability of the powder. Each value reported is an average of three determinations. Determination of powder flowability: The fixed highet cone method was adopted. A cut-stem glass funnel having an internal diameter of $0.6 \mathrm{~cm}$ was used. The funnel was held at $2.5 \mathrm{~cm}$ height (h) over a flat surface. The powder sample (prepared formulae) was allowed to flow gently through the funnel until the cone was formed and reached the funnel surface orifice, powder flow was stopped and the average diameter of the formed cone (D) was determined. The area of base of cone was taken as a measure of internal friction between particles. The angle of repose was calculated by the equation (10): $\tan (\alpha)=2 \mathrm{~h} / \mathrm{D}$. The micromeritic properties were done in triplicate and an average of three determinations was calculated.

\subsubsection{Fourier transform infrared spectroscopy (FTIR)}

An amount of 2-3 mg of dexibuprofen, sodium citrate dihydrate, urea, and prepared formulae were mixed separately with $400 \mathrm{mg}$ of dry potassium bromide powder, compressed into transparent discs and their IR spectra was recorded in the wave length region of $4,000-400 \mathrm{~cm}^{-1}$.

\subsubsection{DSC}

DSC was used to evaluate changes of dexibuprofen characteristic peak and heat enthalpy that might occur after mixing with hydrotropic agents. Thermograms of dexibuprofen, sodium citrate dihydrate, urea, and best formula that showed highest dissolution were recorded using Shimadzu DSC-50 calorimeter. The instrument was calibrated using purified indium (99.99\%). Samples of (2-4 mg) of each substances as well as the best formula were heated under a nitrogen atmosphere as a carrier gas on an aluminum pan at a flow rate $25 \mathrm{~mL} / \mathrm{min}$ and a heating rate of $10^{\circ} \mathrm{C} / \mathrm{min}$ over a temperature range of 20 to $300^{\circ} \mathrm{C}$.

\section{Results and Discussion}

\subsection{Visual inspection}

The prepared formulae were white in color and odorless.

\subsection{Determination of loading efficacy}

The entrapment efficacy (EE) of drug loading in the prepared formulae are presented in Table 2. It ranged from 96.67 to $99.79 \%$ indicating that there is no effect of hydrotropic agents on the drug.

\subsection{Solubility and $p H$}

Table 3 shows the phase solubility study and $\mathrm{pH}$ for $1 \%$ aqueous solution of the prepared formulae. It was noticed that increasing the amount of sodium citrate dihydrate and urea lead to increasing the solubility.

Table 2. Entrapment efficiency of the prepared formulae

\begin{tabular}{lll}
\hline Formula & $\begin{array}{l}\text { Theoretical percent of } \\
\text { dexibuprofen (\%) }\end{array}$ & Entrapment efficiency (\%) \\
\hline F1 & 22.22 & 96.67 \\
F2 & 18.18 & 94.61 \\
F3 & 15.38 & 98.31 \\
F4 & 14.28 & 98.67 \\
F5 & 12.5 & 97.76 \\
F6 & 11.11 & 96.49 \\
F7 & 10.53 & 99.62 \\
F8 & 9.52 & 99.79 \\
F9 & 8.69 & 99.42 \\
\hline
\end{tabular}


Table 3. Phase solubility, pH (1\% solution) and percent mass gain of the prepared formulae

\begin{tabular}{lccc}
\hline Formula & $\begin{array}{l}\text { Solubility }(\mathrm{g} / 100 \mathrm{~mL}) \\
\text { for dexibuprofen in } \\
\text { the prepared formulae }\end{array}$ & $\begin{array}{c}\mathrm{pH} \\
(1 \% \text { solution })\end{array}$ & $\begin{array}{c}\text { Percent mass gain } \\
(\% \mathrm{~m} / \mathrm{m})\end{array}$ \\
\hline F1 & 0.287 & 6.4 & $0.328 \%$ \\
F2 & 0.598 & 6.8 & $0.568 \%$ \\
F3 & 0.619 & 7.0 & $0.357 \%$ \\
F4 & 0.753 & 6.5 & $0.161 \%$ \\
F5 & 1.885 & 6.9 & $0.346 \%$ \\
F6 & 2.247 & 7.1 & $0.421 \%$ \\
F7 & 2.354 & 6.5 & $0.344 \%$ \\
F8 & 3.091 & 6.8 & $0.293 \%$ \\
F9 & 4.182 & 7.8 & $0.431 \%$ \\
\hline
\end{tabular}

This is due to increasing the concentrations of alkali metal salts of various acids (4), and it is concluded that the solubility of dexibuprofen increases synergistically by mixed hydrotropy. The improvement of solubility may be due to changing the crystal forms, different habit, structure and surface modification (11). The $\mathrm{pH}$ for $1 \%$ aqueous solution ranges from 6.4 to 7.8 and that increasing the amount of sodium citrate and urea lead to increasing the $\mathrm{pH}$. The solubility of dexibuprofen increases slightly with an increase in $\mathrm{pH}$, which may be due to the acidic nature of dexibuprofen. Thus, it can be said that, the solubility enhancement of drug by hydrotropes is not entirely due to a $\mathrm{pH}$ effect, but it is largely due to hydrotropy (12).

\subsection{Hygroscopicity}

The $\%$ mass gain of the prepared formulae is presented in Table 3. It ranges from 0.161 to 0.568 so the prepared formulae are described as slightly hygroscopic (7).

\subsection{In vitro dissolution studies}

The dissolution profiles of dexibuprofen from the prepared formulae were done and graphically represented in Figures 1, 2, and 3. Table 4 shows the percent dexibuprofen dissolved from different formulae. Dissolution of drug depends on physicochemical and physicotechnical properties of drug particle. These forms directly affect the absorption kinetics of drug and bioavailability of formulae. This assumes greater importance for a drug exhibiting low solubility that makes absorption to be dissolution rate limited. It is established that the modification of the polymorphic state of a compound can bring an increase in solubility (13). It is obvious that the percent drug dissolved from the prepared formulae containing higher amounts of sodium citrate dihydrate and urea (F7, F8, and F9) (92.46, 92.67, and $97.57 \%$ after $10 \mathrm{~min}$ ) which is higher than the percent drug dissolved from the prepared formulae containing lower amounts of sodium citrate dihydrate and urea (F1, F2, and F3) $(53.47,54.83$, and $65.97 \%$ after $20 \mathrm{~min})$. The improvement of dissolution

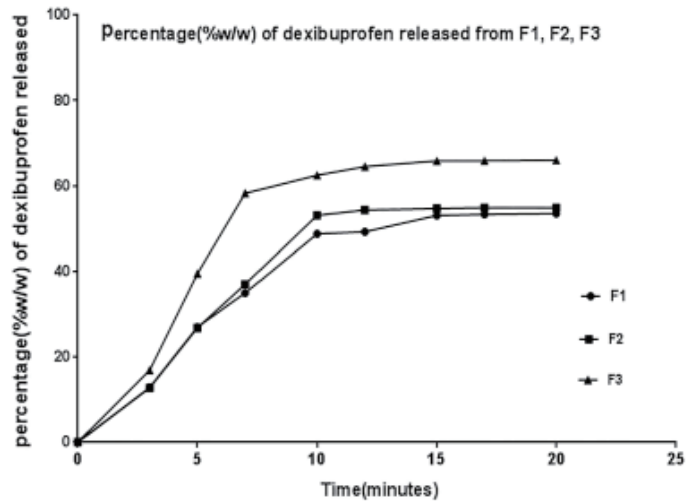

Figure 1. In vitro release profile of dexibuprofen from F1, F2, and $\mathbf{F 3}$ formulae. $500 \mathrm{~mL}$ distilled water was used as dissolution media at $37 \pm 0.5^{\circ} \mathrm{C}$ while maintaining stirring speed at $50 \mathrm{rpm}$. Samples were estimated spectrophotometrically at $\lambda_{\max } 222 \mathrm{~nm}$ for dissolving drug in triplicate and an average of three determinations was calculated.

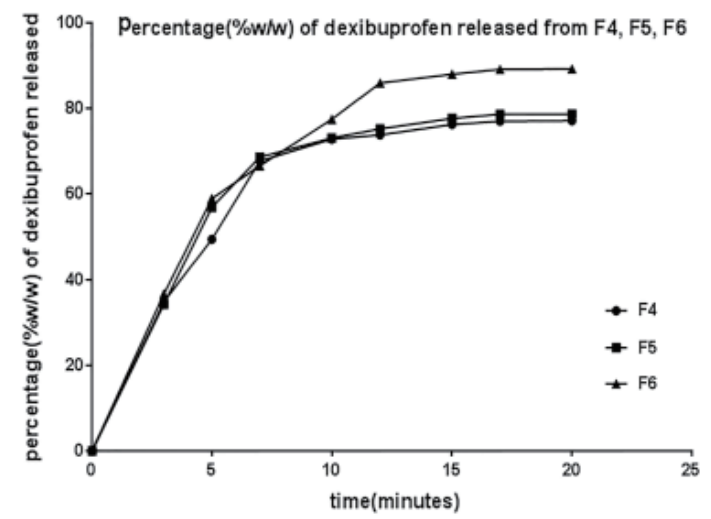

Figure 2. In vitro release profile of dexibuprofen from F4, F5, and $\mathbf{F} 6$ formulae. $500 \mathrm{~mL}$ distilled water was used as dissolution media at $37 \pm 0.5^{\circ} \mathrm{C}$ while maintaining stirring speed at $50 \mathrm{rpm}$. Samples were estimated spectrophotometrically at $\lambda_{\max } 222 \mathrm{~nm}$ for dissolving drug in triplicate and an average of three determinations was calculated.

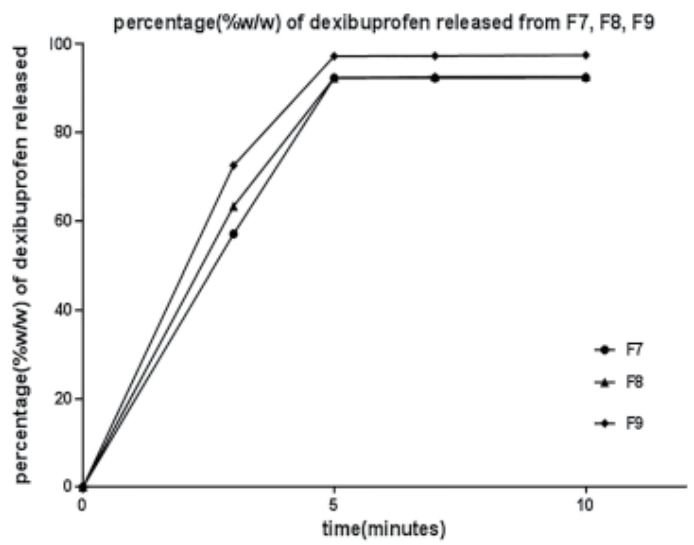

Figure 3. In vitro release profile of dexibuprofen from F7, F8, and F9 formulae. $500 \mathrm{~mL}$ distilled water was used as dissolution media at $37 \pm 0.5^{\circ} \mathrm{C}$ while maintaining stirring speed at $50 \mathrm{rpm}$. Samples were estimated spectrophotometrically at $\lambda_{\max } 222 \mathrm{~nm}$ for dissolving drug in triplicate and an average of three determinations was calculated. 
Table 4. In-vitro release of dexibuprofen from the prepared formulae

\begin{tabular}{|c|c|c|c|c|c|c|c|c|}
\hline \multirow[b]{2}{*}{ Formula } & \multicolumn{8}{|c|}{ Percentage $(\% \mathrm{w} / \mathrm{w})$ of dexibuprofen released after the following time intervals in minutes } \\
\hline & 3 & 5 & 7 & 10 & 12 & 15 & 17 & 20 \\
\hline $\mathrm{F} 1$ & 12.82 & 26.97 & 34.95 & 48.75 & 49.23 & 53.04 & 53.3 & 53.47 \\
\hline $\mathrm{F} 2$ & 12.70 & 26.8 & 36.99 & 53.1 & 54.39 & 54.64 & 54.83 & 54.83 \\
\hline F3 & 16.82 & 39.45 & 58.24 & 62.46 & 64.49 & 65.81 & 65.85 & 65.97 \\
\hline F4 & 34.85 & 49.49 & 67.74 & 72.86 & 73.83 & 76.28 & 77.02 & 77.19 \\
\hline F5 & 34.22 & 56.93 & 68.66 & 73.12 & 75.26 & 77.72 & 78.68 & 78.71 \\
\hline F6 & 36.64 & 59 & 66.64 & 77.52 & 85.98 & 88.03 & 89.14 & 89.24 \\
\hline F7 & 57.14 & 92.36 & 92.38 & 92.46 & & & & \\
\hline F8 & 63.4 & 92.43 & 92.6 & 92.67 & & & & \\
\hline F9 & 72.63 & 97.33 & 97.36 & 97.57 & & & & \\
\hline
\end{tabular}

Table 5. Micromeritic properties of the prepared formulae

\begin{tabular}{|c|c|c|c|c|c|c|c|c|}
\hline Formula & $\begin{array}{l}\text { Bulk volume } \\
\quad(\mathrm{mL})\end{array}$ & $\begin{array}{c}\text { Tapped volume } \\
(\mathrm{mL})\end{array}$ & $\begin{array}{l}\text { Bulk density } \\
\quad(\mathrm{g} / \mathrm{mL})\end{array}$ & $\begin{array}{c}\text { Tapped density } \\
(\mathrm{g} / \mathrm{mL})\end{array}$ & Hausner ratio & $\begin{array}{c}\text { Compressibility } \\
(\%)\end{array}$ & $\operatorname{Tan} \theta$ & $\begin{array}{l}\text { Angle of repose } \\
\text { (degrees) }\end{array}$ \\
\hline F1 & 228 & 202 & 0.44 & 0.50 & 1.13 & 11.40 & 0.50 & 31.30 \\
\hline $\mathrm{F} 2$ & 227 & 198 & 0.44 & 0.51 & 1.15 & 12.78 & 0.54 & 34.42 \\
\hline F3 & 223 & 197 & 0.45 & 0.51 & 1.13 & 11.66 & 0.50 & 31.30 \\
\hline F4 & 233 & 207 & 0.43 & 0.48 & 1.13 & 11.16 & 0.51 & 32.27 \\
\hline F5 & 235 & 208 & 0.43 & 0.48 & 1.13 & 11.49 & 0.53 & 33.26 \\
\hline F6 & 224 & 199 & 0.45 & 0.50 & 1.13 & 11.16 & 0.51 & 32.27 \\
\hline F7 & 229 & 203 & 0.44 & 0.49 & 1.13 & 11.35 & 0.50 & 31.30 \\
\hline F8 & 222 & 196 & 0.45 & 0.51 & 1.13 & 11.71 & 0.51 & 32.27 \\
\hline F9 & 225.5 & 200.5 & 0.44 & 0.50 & 1.12 & 11.09 & 0.50 & 31.30 \\
\hline
\end{tabular}

behavior could be attributed to solubilization of the drug using hydrotropic agents (8). The faster dissolution could be due to better solubility of the prepared formulae that contains higher amounts of hydrotropic agents because increasing the amount of hydrotropic agents in the formulae leads to increased solubility and dissolution rate.

\subsection{Micromeritic properties}

The results of micromeretic properties of the prepared formulae are shown in Table 5. Bulk densities ranged from 0.43 to $0.45 \mathrm{~g} / \mathrm{mL}$ and tapped densities ranged from 0.48 to $0.51 \mathrm{~g} / \mathrm{mL}$. Hausner ratio values ranged from 1.13 to 1.15 indicating low to moderate interparticle friction and thus good flow properties (14). They showed good flow with respect to average \% compressibility which ranged from (11.09 to $11.71 \%$ ). Also, the angle of repose ranged from $31.30^{\circ}$ to $34.42^{\circ}$ indicating good flow properties (15).

\subsection{Fourier transform infrared spectroscopy (FTIR)}

The collected FTIR of plain dexibuprofen drug, sodium citrate dihydrate, urea and the prepared formulae are shown in (Figure 4). The main peaks characteristic of the drug are identical and well identified in the prepared formulae. This can be summarized: prominent peaks at $3,087,1,707,1,406,1,050 \mathrm{~cm}^{-1}$ corresponding to $\mathrm{O}-\mathrm{H}$ stretching, $\mathrm{C}=\mathrm{O}$ stretching, $\mathrm{C}-\mathrm{C}$ stretching, $\mathrm{O}-\mathrm{H}$ bending respectively. The FTIR showed no changes occurred in the chemical nature of drug indicating absence of chemical interaction between drug and hydrotropic agents and so, confirming drug stability. From these results, it can be speculated that a drughydrotropic agent hydrogen bond existed in this formula and caused reduced drug recrystallization (16), also intermolecular interaction between drug and hydrotropic agents occurred $(5,6)$.

\section{8. $D S C$}

DSC pattern of plain dexibuprofen, sodium citrate dihydrate, urea and the F9 formula are recorded in Figure 5. It is clear that, the DSC of plain dexibuprofen drug showed an endothermic peak at onset of $47.25^{\circ} \mathrm{C}$ reaching a peak at $56.51{ }^{\circ} \mathrm{C}$, an endset of $66.49^{\circ} \mathrm{C}$ and the enthalpy was $210.40 \mathrm{~J} / \mathrm{g}$ while hydrotropic agents; sodium citrate and urea showed an endothermic peak at $169.8^{\circ} \mathrm{C}$ and $138.53^{\circ} \mathrm{C}$, respectively. The endothermic peak of drug is related to its melting point. There was a slight difference in melting endotherms of selected formulae as well as decreasing intensity and loss of sharpness compared to that of pure drug (drug $=62.52^{\circ} \mathrm{C}$, sodium citrate dehydrate $=143.65^{\circ} \mathrm{C}$, urea $=103.05^{\circ} \mathrm{C}$ ). These results might be explained in terms of a presence of intermolecular interaction between drug and hydrotropic agents. Basically the thermal process of any mixture is the sum of individual components. However, there was invariably very little 


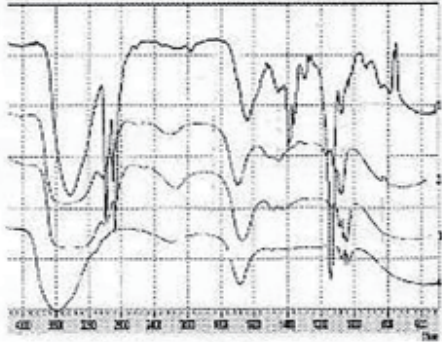

(a)

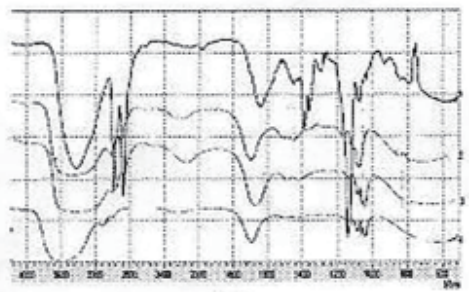

(d)

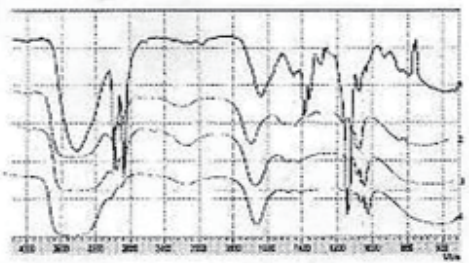

(g)

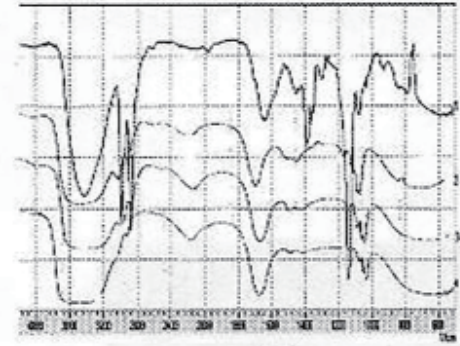

(b)

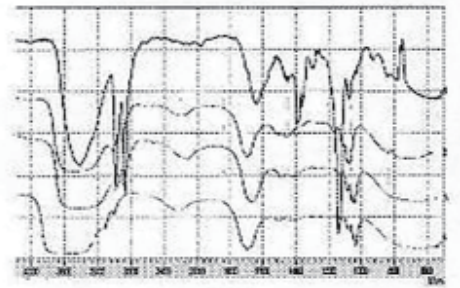

(e)

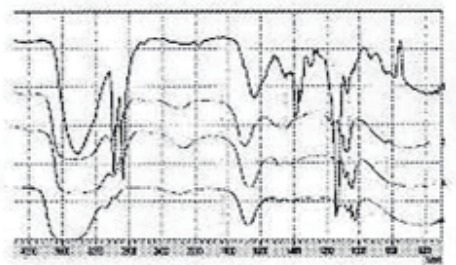

(h)

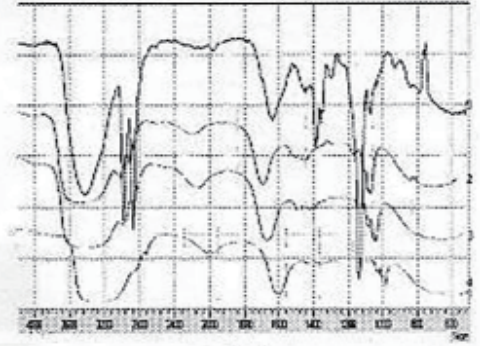

(c)

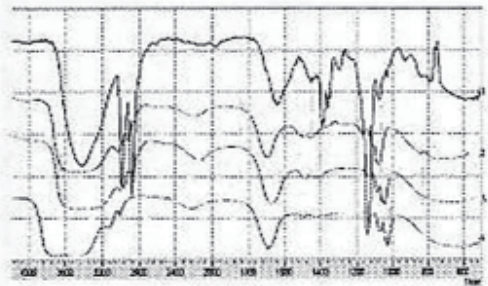

(f)

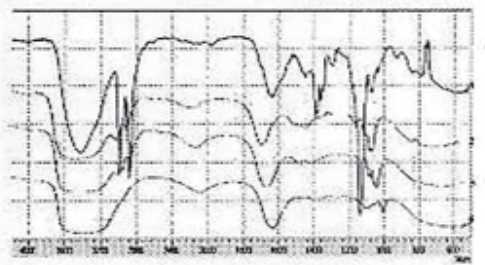

(i)

Figure 4. IR spectroscopy for dexibuprofen (1), sodium citrate dihydrate (2), urea (3) and formulae (4). An amount of 2-3 $\mathrm{mg}$ of dexibuprofen, sodium citrate dihydrate, urea, and prepared formulae were mixed separately with $400 \mathrm{mg}$ of dry potassium bromide powder, compressed into transparent discs and their IR spectra was recorded in the wave length region of 4,000-400 $\mathrm{cm}^{-1}$. (a) IR spectroscopy for dexibuprofen, sodium citrate dihydrate, urea, and F1. (b) IR spectroscopy for dexibuprofen, sodium citrate dihydrate, urea and F2. (c) IR spectroscopy for dexibuprofen, sodium citrate dihydrate, urea, and F3. (d) IR spectroscopy for dexibuprofen, sodium citrate dihydrate, urea, and F4. (e) IR spectroscopy for dexibuprofen, sodium citrate dihydrate, urea, and F5. (f) IR spectroscopy for dexibuprofen, sodium citrate dihydrate, urea and F6. (g) IR spectroscopy for dexibuprofen, sodium citrate dihydrate, urea, and F7. (h) IR spectroscopy for dexibuprofen, sodium citrate dihydrate, urea, and F8. (i) IR spectroscopy for dexibuprofen, sodium citrate dihydrate, urea, and F9.

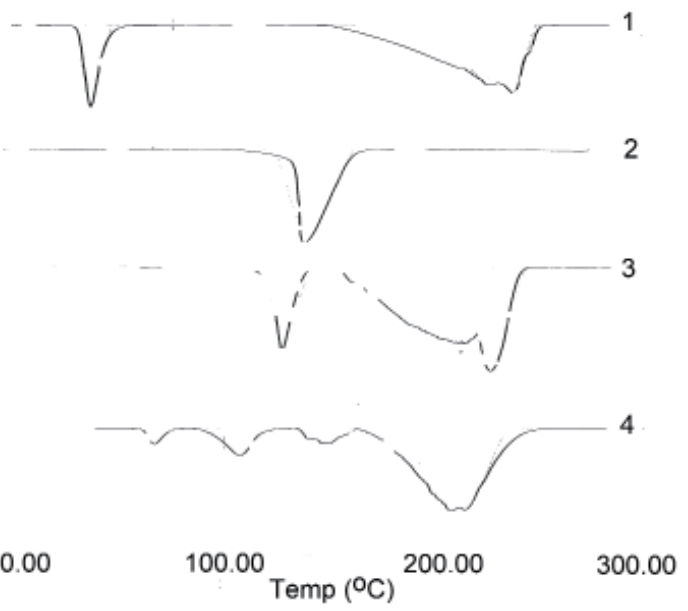

Figure 5. DSC for dexibuprofen, sodium citrate dihydrate, urea, and F9 formula. (1) dexibuprofen, (2) sodium citrate dihydrate, (3) urea, (4) F9. The instrument was calibrated using purified indium $(99.99 \%)$. Samples of (2-4 mg) of each substance as well as the best formula were heated under a nitrogen atmosphere as a carrier gas on an aluminum pan at flow rate $25 \mathrm{~mL} / \mathrm{min}$ and a heating rate of $10^{\circ} \mathrm{C} / \mathrm{min}$ over a temperature range of 20 to $300^{\circ} \mathrm{C}$. change in transition temperature when mixing two or more components. When two substances are mixed, the purity of each may be reduced and generally very slight lower melting endotherms may result (17). This explains the very slight shift drug hydrotropic agent peak. Previous work (18), accepts small events and considers them to not represent any determinable interaction. This could be due to differences in moisture content of samples (19). Also, some changes in the peak shape and height to width ratio can be seen because of possible differences in the mixture sample geometry (20). The observed change in enthalpy indicates a change in crystallinity of the drug (21), resulting in an increase in dissolution rate.

In conclusion, the mixed hydrotropic solubilization technique significantly influenced solubilization of dexibuprofen which is practically insoluble thus contributing to dissolution improvement. Increasing the amount of hydrotropic agents leads to an increase in $\mathrm{pH}$, solubility, rate and amount of dexibuprofen release from the dissolution medium. The highest dissolution 
rate was achieved from F9 formula at drug: hydrotropic agent ratio 1:3:7.5. The prepared formulae are slightly hygroscopic and show good flow properties. Drug stability with hydrotropic agents and intermolecular interaction between them has been confirmed by FTIR and DSC results.

\section{Acknowledgements}

This work was supported by a grant from National Organization of Drug Control and Research and Department of Pharmaceutical Technology, Faculty of Pharmacy, Tanta University.

\section{References}

1. Adams SS, Bresloff P, Mason CG. Pharmacological differences between the optical isomers of ibuprofen evidence for metabolic inversion of the (-) isomer. J Pharm Pharmacol. 2004; 28:256-257.

2. Bomabello A, Galmozzi MR, Canaparo R, Isaia GC, Serepe L, Muntoni E. Dexibuprofen ( $(++)$-Isomer Ibuprofen) reduces gastric damage and improves analgesic anti-inflammatory effects in rodents. Anesth Analg. 2003; 97:402-408.

3. Amit K, Mahalaxmi RP, Srinivas, Deepak K. Enhancement of solubility and dissolution of poorly soluble drug: Ketoprofen as a model drug. J Chem Pharm Res. 2011; 3:268-276.

4. Neuberg C. Hydrotropy. Biochem Z. 1961; 76:107-109.

5. Maheshwari RK. Analysis of frusemide by application of hydrotropic solubilization phenomenon. Indian Pharmacist. 2005; 4:55-58.

6. Maheshwari RK. Mixed hydrotropy in spectrophotometric analysis of poorly water-soluble drug. Indian Pharmacist. 2007; 6:66-67.

7. Niazi S. Physicochemical properties affecting bioequivalence. In: Handbook of Bioequivalence Testing. Informa Healthcare, Mortimer Street, London, UK, 2007; pp. 181-182.

8. Rajesh K, Rahul Nair, Rajalakshmi R, Latha P, Arun kumar KS, Nama V. Inclusion complex of dexibuprofen and its in-vitro and in-vivo evaluation. Int Bio Pharm Res. 2011; 2:34-38.

9. Aulton ME. Pharmaceutics. The science of dosage form design (Taylor k, ed.). Churchill Livingstone, London, UK, 2002; p. 197.

10. Bastos MO, Friedrich RB, Beck CR. Effects of fillerbinders and lubricants on physicochemical properties of tablets obtained by direct compression: A 22 Factorial Design. Lat Am J Pharm. 2008; 27:578-583.

11. Badawy AA, El-Nabarawwy MA, El-Houssieny BM, Kamel MM. Formulation and evaluation of albendazole solid dispersion. J Drug Res. 2011; 32:11-21.

12. Rajesh KM, Arpna I. Formulation and evaluation of aceclofenac injection made by mixed hydrotropic solubilization technique. Iran J Pharm Res. 2010; 9:233242.

13. El-Houssieny BM, Soliman II, Mansour MM. Preparation and evaluation of etodolac spherical agglomerate crystals prepared by the quasi emulsion solvent diffusion method. Bull Fac Pharm Cairo Univ. 2009; 47:85-102.

14. Shahi SR, Agrawa GR, Shinde NV, Shaikh SA. Formulation and in-vitro evaluation of orodispresible tablets of etoricoxib with emphasis on comparative functionality evaluation of three classes of superdisintegrants. Russ J Chem. 2008; 1:292-300.

15. European Pharmacopoeia. Sixth Edition. Council of Europe, 67075 Strasbourg Cedex, France, 2007; pp. 320321.

16. Silverstein RM. Spectrophotometric Identification of Organic Compounds. John Wiley and Sons, New York, USA, 1981; p. 112.

17. Botha SA, Lotter AP. Compatibility study between atenolol and tablet excipient using differential scanning calorimetery. Drug Dev Ind Pharm. 1990; 19:235-258.

18. Wells JI. Pharmaceutical Preformulation. The physicochemical properties of drug substances. Ellis Horwood Ltd, Chichester, UK, 1988; pp. 86-102.

19. Biliaderis CG. Structures and phase transitions of starch in food systems. Food Tech. 1992; 46:98-109.

20. Duvall RN, Koshy KT, Dashiell RE. Comparative evaluation of dextrose and spray dried lactose in direct compression system. Int J Pharm Sci. 1965; 54:11961200.

21. Ford JL, Stewart AF, Dobois JL. The properties of solid dispersion of indomethacin or phenylbutazone in polyethylene glycol. Int J Pharm. 1986; 28:11-22.

(Received April 7, 2014; Revised August 13, 2014; Accepted August 22, 2014) 